\title{
DESIGN AND IMPLEMENTATION OF A DMD BASED VOLUMETRIC 3D DISPLAY
}

\author{
Veysel Yücesoy, Doruk Tunaoğlu, Metodi Kovachev, Rossitza Ilieva, Levent Onural
}

Department of Electrical and Electronics Engineering, Bilkent University, TR-06800, Ankara, Turkey

\begin{abstract}
A real image swept-volume volumetric display is developed. A piston type moving screen is used to obtain the desired volume. A commercially available DMD device is used to project $2 \mathrm{D}$ slices of a $3 \mathrm{D}$ frame. There is a varying magnification effect during the projection because of the optical design of the system; raw 3D video frames are processed by a software to generate the appropriate $2 \mathrm{D}$ slices by also correcting the magnification. Synchronization between the hardware and the software is achieved via a microcontroller. The overall system is capable of printing 12 3D frames per second where a 3D frame consists of 90 2D slices with a resolution varying from $512 \times 512$ to 450x450 (approx. 20M voxels per one 3D frame). Although some flickering effect is observed due to rather low $3 \mathrm{D}$ frame rate, results are visually satisfactory.
\end{abstract}

Index Terms - Displays, three-dimensional displays, volumetric displays

\section{INTRODUCTION}

Many different types of display systems are reported in the literature. [2 - 7] In general, it is possible to classify 3D displays into three groups [1] :

- Autostereoscopic displays

- Volumetric displays

- Holographic displays

Volumetric displays may be further classified as virtual image and real image displays [1]. Real image displays include swept volume and static volume displays [1]. Our display design is also a real image swept-volume volumetric display.

In this type of display, the whole $3 \mathrm{D}$ image is reproduced by projecting $2 \mathrm{D}$ slices of the $3 \mathrm{D}$ scene at a sufficient rate for continuous perception. For example,

\footnotetext{
This work is supported by European Commission Sixth Frame Program under the grant no: 511568 with the acronym 3DTV.
}

Favalora et al. [2] developed such a system using a semicircle projection screen which rotates at a high speed. They projected 200 slices each having a resolution of 768 by 768 pixels to form a 3D image. This idea is used and developed by Actuality System to create Perspecta [3].

There is another swept-volume rotating screen type display, designed by Holoverse, [4], [5], [6], in which designers claim to have $1024 \times 768 \times 360$ effective resolution and increased color depth.

Another example of swept volume displays is the Felix [7], in which a helical sweeping surface and lasers are used to create a 3D scene. This type of a screen was also among our possible choices, however, due to various reasons, we decided to use another form.

Our design puts together desired features of these examples and uses commercially available parts. We used a commercially available DMD chip to project 2D slices like in Holoverse's design, but we used a separate piston type screen instead of Felix's helical one for reasons explained below.

Hardware and software designs of the display are introduced in Section 2. Results and conclusion are given in Section 3.

\section{DESIGN OF THE VOLUMETRIC DISPLAY}

\subsection{Hardware}

The first step in hardware design was the choice of the plane which will cover the active volume in the display. We conducted some preliminary experiments and tried to see the advantages and disadvantages of some possible different structures. As rotating planes, we tried three different structures: a simple propeller with an inclination of 45 degrees as shown in Figure 1.a and b, a simple rotating rectangular plane as shown in Figure 1.c and a rotating helix. We observed that the first two structures resulted in some undesired uneven variation of the usable depth as shown in Figure 1.d.

Even the helix was a desirable choice; it still had some depth-related problems at its center. However, since a helix has a symmetric shape with respect to its rotation axis, rotations would become smoother and there would be fewer vibrations then other structures. 
We also tried a piston type screen which goes back and forth by a DC motor and sweeps a cylindrical volume. A problem in such a design is the vibrations due to conversion of rotational motion to linear motion to move the screen: the load on the motor is not symmetrically distributed so vibrations are inevitable. Since it was possible to reduce these vibrations by some structural solutions, we decided to use a piston type screen in our volumetric display project due to its advantages as outlined above.

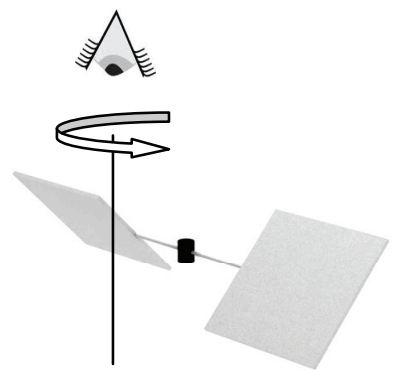

a

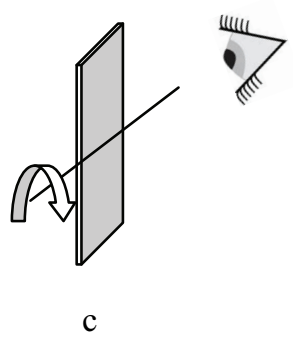

Figure 1: a. Propeller design (front view), b. Propeller design (side view), c. Rectangular design and d. Resultant volume for the rectangular design

The swept volume of the display had $9 \mathrm{~cm}$ depth in total. The main idea behind our display design was simple: $2 \mathrm{D}$ vertical slices of a 3D object will be prepared by software and they will be projected on the sweeping 2D plane in a synchronous manner. If the display will be able to achieve a speed faster than the human perception, a good 3D image will be obtained in the swept volume. Losses in the speed of the overall system up to some critical point will only cause some flickering images, however, any problem in the synchronization of the sweeping plane and reflecting device will cause very serious perception problems and images may even become meaningless. The DC motor in our project was able to yield approximately 5-6 rotations per second due to air friction of the screen and the physical structure of the metal parts. 6 rotations per second resulted in 12 sweeps of the 2D plane per second. We decided to use $1 \mathrm{~mm}$ resolution in depth as a design parameter; which implies that we needed 90 different 2D slices in 1 sweep of the $3 \mathrm{D}$ volume. Also a progressive scan technique was used where the same $2 \mathrm{D}$ slice is written twice: once when the screen is going forward and once again in the same location when it is moving backward. Considering all these calculations, it was clear that a reflector that can handle more then 1080 images per second and that can be fully synchronized with the moving screen system was required in this project. We decided to use a commercially available DMD device which can yield such rates in binary mode.

Synchronization was achieved by using an encoder disc with 360 slits, which is attached to shaft and an optical incremental encoder.

These encoders have two separate channels with a phase difference and it is possible to get all the information about the shaft (i.e. rotation direction, rotation position, rotation speed, etc.) when these channels are properly connected to a microcontroller. A sample channel pair which can be traced by a microcontroller to understand how fast and to which position the motor is rotating, is shown in Figure 2.

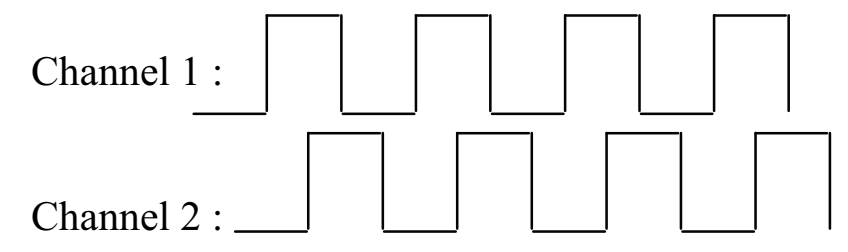

Figure 2 : Encoder channel timing

The microcontroller, in this case, was also used to trigger the DMD when predefined positions are reached by the motor shaft. This trigger signal was a simple impulse of duration of 1 dot of the encoder disc.

A problem was the magnification because of the optical system that we used in front of the DMD. Due to the used objective lens it was possible to focus the image on only one depth. This was the center depth in our case. Since the focal depth of the objective lens was larger then the total depth of our swept volume $(\sim 13 \mathrm{~cm}$ and $\sim 9 \mathrm{~cm}$, respectively) we did not have focusing problems. However, we had some magnification problems because the same image was printed larger on the screen when it is in a further position. This magnification effect was corrected by calibrating the image size, using software.

Another interesting problem in our design was about the conversion of the rotational motion to linear motion. Since we got the position information directly from the shaft, we had information about the rotational motion. However, the important motion for our timing and synchronization was the linear motion so the link between these two should be as precise as possible. We concluded to use an arc-cosine mapping between rotational intervals and linear intervals to be able to catch equally spaced linear intervals. 


\subsection{Software}

Using a piston type screen to obtain a 3D object with good resolution in depth, we needed a device which had a very high frame per second rate. For instance, for projecting a 3D frame with 90 2D slices with 20 3D frames per second rate our device must print 1800 2D frames per second. Because of that reason using a DMD device is a good choice since it can go up to $8 \mathrm{~K}$ frames per second when it is used in binary mode. Software design is divided into three parts: Controlling the DMD device in a proper way and adjusting its settings was the first part. Then raw 3D video is processed in order to obtain an appropriate 3D image with the optical system. Finally microcontroller is programmed so that $2 \mathrm{D}$ slices are printed in synchronization with the position of the screen.

The first part handles the DMD device using its provided library and achieves tasks like memory allocation, uploading of the $2 \mathrm{D}$ frames to the device, external trigger setup, etc. Also other small details like setting the dark frame time (time between the end of a frame and the beginning of the next) to zero or taking the mirror image to adjust for the changes in optical system are handled in this part.

As explained in the hardware section there is a magnification problem because of the optical system. Even if we write the same image on the DMD, it is magnified linearly as the screen goes away. So we came up with a simple solution: First we brought the screen to its closest point to the DMD. Then using software we fit the biggest square on to the screen and marked the edges and center of it. Then we brought the screen to its furthest position and tried to achieve the same square on the marked points. As a natural result, smaller resolution is obtained when the screen is in its furthest position. For our setup, resolution was decreased from $512 \times 512$ to $450 \times 450$ linearly. Since decimation is easier than interpolation we decided to work on $3 \mathrm{D}$ videos with a size $512 \times 512 \times 90$. We decided to use the following simple decimation algorithm that works well with simple geometric shapes. First we calculate the resolution of the current $2 \mathrm{D}$ slice considering the linear magnification. Then whenever we see a black pixel in the 2D slice of the raw binary high-resolution video, we write a corresponding black pixel on the low-resolution 2D slice.

We complete the projection of a 3D frame by projecting the $902 \mathrm{D}$ slices in the normal order when the piston moves back; and then we project the same 90 2D slices in the reverse order as the piston comes forward. Because of hardware limitations we can project $123 \mathrm{D}$ frames per second where the size of each 3D frame is $512 \times 512 \times 90$.

Final step in the software design is the implementation of the microcontroller's tasks. The function of the microcontroller is to measure the position of the motor and to trigger the DMD at the correct positions. Position measurement was achieved by observing the channels of the encoder. As explained in the hardware section, the position of the screen is equal to the arc-cosine of the motor shaft position and the DMD is triggered accordingly.

\section{RESULTS AND CONCLUSION}

Our display had a very good resolution $(512 \times 512 \times 90)$ and visual quality. The best quality is observed when we look exactly towards the screen. However, as we increase the angle of observation the quality degrades but is still quite satisfactory. As in all volumetric displays, we see transparent images. And when the angle of observation is more than 90 degrees or less than -90 degrees nothing can be seen. Even though there are disadvantages we are still satisfied with the performance of our volumetric display as shown in Figure 3.

\section{REFERENCES}

[1] P. Benzie, J. Watson, P. Surman, I. Rakkolainen, K. Hopf, H. Urey, V. Sainov, and C. von Kopylow, "A Survey of 3DTV Displays: Techniques and Technologies", in IEEE Transactions on Circuits and Systems for Video Technology, vol. 17, no. 11, pp. 1647-1658, November 2007.

[2] G. E. Favalora, R. K. Dorval, D. M. Hall, M. Giovinco, and J. Napoli, "Volumetric 3-D display system with rasterization hardware", in Proc. SPIE, Stereoscop. Displ. Virt. Real. Syst. VIII, vol. 4297, pp. 227-235, 2001.

[3] M. Thomas, R. K. Dorval, J. L. Bareau, "Volumetric three-dimensional display system”, U.S. Patent 6554 430, Apr. 29, 2003.

[4] D. J. Solomon, "Visual Special Effects Displ. Device", U.S. Patent 6404 409, Jun. 11, 2002.

[5] D. J. Solomon, "Three-dimensional volumetric display system”, U.S. Patent 4983 031, Jan. 8, 1981.

[6] D. J. Solomon, "High contrast display device enclosure system using transparent light polarizing means", U.S. Patent 4974 946, Jan. 6, 1998.

[7] D. Bahr, K. Langhans, M. Gerken, C. Vogt, D. Bezecny, and D. Homann, "Felix: A volumetric 3D laser display", in Proc. SPIE Projection Displ. II, San Jose, CA, vol. 2650, pp. 265-273, 1996. 


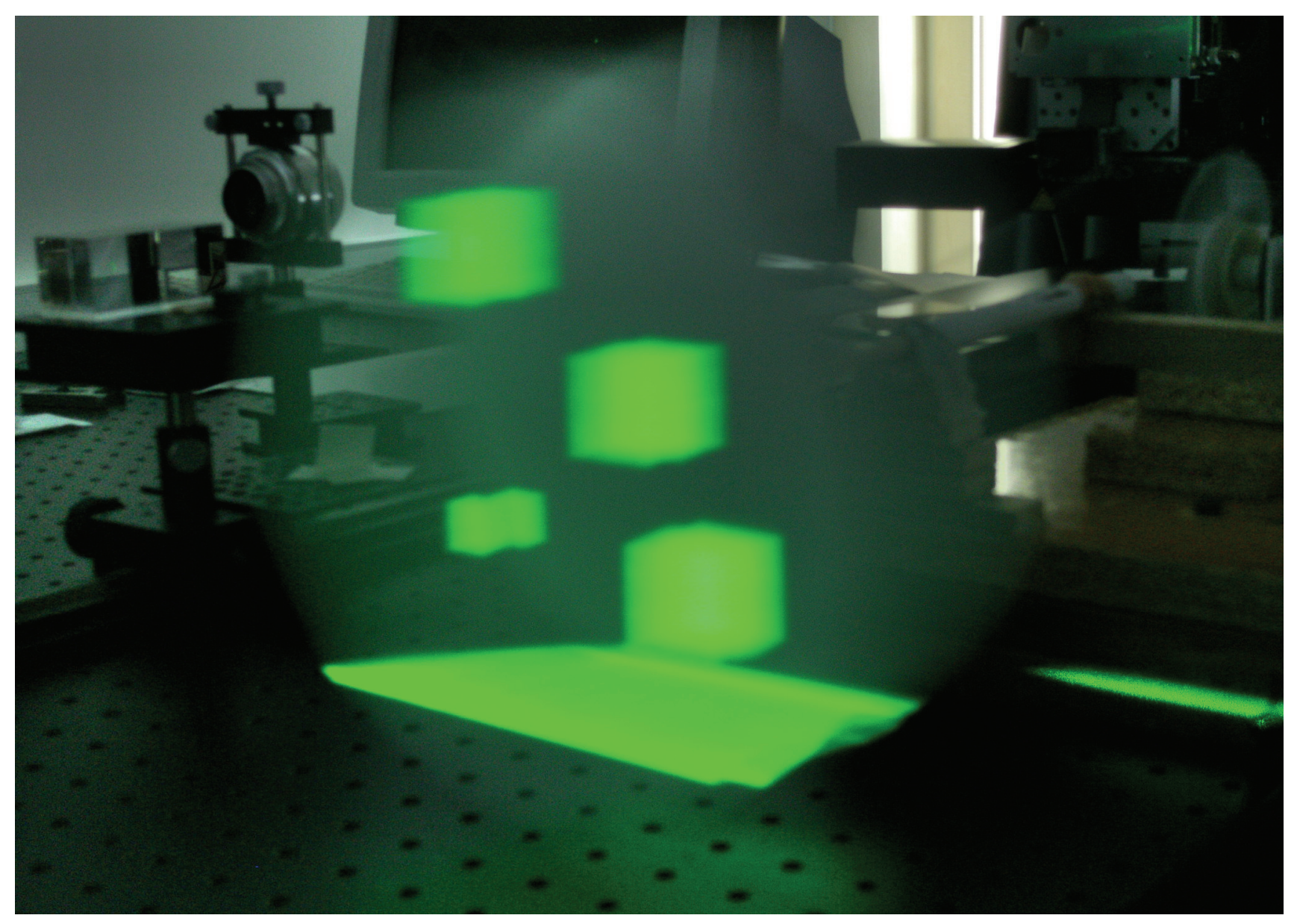

Figure 3: The designed volumetric display in operation. 